\title{
SjCa8, a calcium-binding protein from Schistosoma japonicum, inhibits cell migration and suppresses nitric oxide release of RAW264.7 macrophages
}

\author{
Ji Liu ${ }^{1,2 \dagger}$, Tong Pan ${ }^{1,2 \dagger}, X_{\text {u You }}^{1,2 \dagger}$, Yiyue $X^{1,2}$, Jinyi Liang ${ }^{1,2}$, Yanin Limpanont ${ }^{3}, X_{i}$ Sun $^{1,2}$, Kamolnetr Okanurak $^{3}$,
} Huanqin Zheng ${ }^{1,2}$, Zhongdao $\mathrm{Wu}^{1,2}$ and Zhiyue LV $\mathrm{V}^{1,2^{*}+}$

\begin{abstract}
Background: Schistosomiasis is considered second only to malaria as the most devastating parasitic disease in tropical countries. Schistosome cercariae invade the host by penetrating the skin and migrate though the lungs and portal circulation to their final destination in the hepatic portal system and eventually the mesenteric veins. Previous studies have shown that the cytotoxic pathways that target schistosomulum in the lung-stage involve nitric oxide (NO) produced by macrophages. By contrast, skin-stage schistosomulas can evade clearance, indicating that they might be freed from macrophage NO-mediated cytotoxicity to achieve immune evasion; however, the critical molecules and mechanisms involved remain unknown.

Methods: Recombinant SjCa8 (rSjCa8), an 8-kDa calcium-binding protein that is stage-specifically expressed in cercaria and early skin-stage schistosomulas of Schistosoma japonicum, was incubated with mouse RAW264.7 macrophages. Effects on macrophage proliferation were determined using Cell Counting Kit-8. Next, transwell assay was carried out to further investigate the role of $\mathrm{rSjCa8}$ in macrophage migration. The effects of $\mathrm{rSjCa8}$ on macrophage apoptosis were evaluated using confocal microscopy and flow cytometry. Additional impacts of rSjCa8 on NO release by lipopolysaccharide (LPS)-stimulated macrophages as well as the underlying mechanisms were explored using fluorescent probe, nitric oxide signaling pathway microarray, quantitative real-time PCR, mutagenesis, and neutralizing antibody approaches.

Results: rSjCa8 exhibited a striking inhibitory effect on macrophage migration, but did not markedly increase cell proliferation or apoptosis. Additionally, rSjCa8 potently inhibited NO release by LPS-stimulated macrophages in a dose- and time-dependent manner, and the inhibitory mechanism was closely associated with intracellular $\mathrm{Ca}^{2+}$ levels, the up-regulation of catalase expression, and the down-regulation of the expression of 47 genes, including Myc, Gadd45a, Txnip, Fas, Sod2, Nos2, and Hmgb1. Vaccination with rSjCa8 increased NO concentration in the challenging skin area of infected mice and reduced the number of migrated schistosomula after skin penetration by cercariae. (Continued on next page)
\end{abstract}

\footnotetext{
* Correspondence: Ivzhiyue@mail.sysu.edu.cn

${ }^{\dagger}$ Equal contributors

'Zhongshan School of Medicine, Sun Yat-sen University, 74 2nd Zhongshan

Road, Guangzhou 510080, China

${ }^{2}$ Key Laboratory for Tropical Diseases Control of Ministry of Education, Sun

Yat-sen University, Guangzhou 510080, China

Full list of author information is available at the end of the article
}

\section{Biomed Central}

(c) 2015 Liu et al. Open Access This article is distributed under the terms of the Creative Commons Attribution 4.0 International License (http://creativecommons.org/licenses/by/4.0/), which permits unrestricted use, distribution, and reproduction in any medium, provided you give appropriate credit to the original author(s) and the source, provide a link to the Creative Commons license, and indicate if changes were made. The Creative Commons Public Domain Dedication waiver (http://creativecommons.org/publicdomain/zero/1.0/) applies to the data made available in this article, unless otherwise stated. 
(Continued from previous page)

Conclusions: Our findings indicate that SjCa8 might be a novel molecule that plays a critical role in immune evasion by S. japonicum cercaria during the process of skin penetration. The inhibitory impacts of rSjCa8 on macrophage migration and $\left[\mathrm{Ca}^{2+}\right]_{\mathrm{i}}$-dependent NO release suggest it might represent a novel vaccine candidate and chemotherapeutic target for the prevention and treatment of schistosomiasis.

Keywords: SjCa8, Schistosoma japonicum, Immune evasion, Macrophage, NO, Calcium-binding protein

\section{Background}

Schistosomiasis, a parasitic zoonosis caused by schistosomes that parasitize the portal and mesenteric veins of the host, remains the second most common epidemic tropical disease worldwide, as this parasite infects 200 million individuals in 73 countries in Asia (including China), Africa, and Latin America, and is responsible for $\sim 100,000$ deaths annually $[1,2]$. Schistosome is a multicellular organism with a complex life cycle, in which a sophisticated immunoevasion system impedes the development of a protective immune response [3, 4]. Because praziquantel represents the only effective drug against schistosomiasis, there is a great risk of the development of drug resistance [5], which represents a severe challenge in the field of schistosomiasis prevention and eradication.

The cercaria stage is a transient free stage in the $S$. japonicum life cycle, and cercaria invasion initiates the process of infection in its definitive host. Although cercariae are relatively simple and short-lived organisms, it is well recognized that the ultraviolet-ray (UV) attenuated cercariae can induce high and stable protection against Schistosoma mansoni challenge in many animal models $[6,7]$. Furthermore, in contrast with most lungstage schistosomula that are eliminated by macrophage NO-mediated cytotoxicity [8, 9], skin-stage schistosomulas can evade clearance by immune cells by penetrating the host's skin surface, indicating that a specific schistosome-derived molecule might exist that facilitates immunoevasion [10].

Our previous studies have shown that $\mathrm{SjCa} 8$, an $8-\mathrm{kDa}$ calcium-binding protein derived from $S$. japonicum, is specifically expressed in cercariae and skin-stage schistosomula, but is silenced in eggs, lung-stage schistosomula, and adult worms [11]. Additionally, previous studies have localized $\mathrm{SjCa} 8$ to the cercarial head gland, penetration gland, secretions, and tegument (where cercariae directly contact and interact with host cells) [11], which suggest that stagespecific expression of $\mathrm{SjCa} 8$ could play a role in the skin penetration process by cercariae, although the mechanisms involved remain unclear. Therefore, in this study, we characterized the effects of $\mathrm{rSjCa} 8$ on macrophage proliferation, apoptosis, migration, and NO release.

\section{Methods}

\section{Preparation of recombinant proteins}

Purified recombinant SjCa8 (rSjCa8) and Sj13 (rSj13, control protein) were prepared as described previously [12] and subsequent endotoxin removal from purified proteins was performed using Detoxi-Gel Endotoxin Removal Columns (Thermo Fisher Scientific, Waltham, MA, USA), according to the manufacturer's instructions [13].

To prepare recombinant mutant $\mathrm{SjCa} 8(\mathrm{rmuSjCa} 8)$, full-length (210 bp) muSjCa8 DNA with site-specific mutations of glutamate (E, positions 26 and 62 ) in the $\mathrm{Ca}^{2+}$-binding loops, which are thought to be key residues for calcium binding $[11,14]$, to glutamine $(\mathrm{Q})$, was synthesized by Takara Bio Inc. (Dalian, China). The mutant gene was subsequently cloned, expressed, purified, and subjected to endotoxin removal following established procedures [11, 13].

\section{Cell culture}

The mouse RAW264.7 macrophage-like cell line was obtained from the American Type Tissue Culture Collection (Rockville, MD, USA), cultured in Dulbecco's Modified Essential Medium (DMEM, Gibco ${ }^{\circ}$ Life Technologies, Grand Island, NY, USA) supplemented with $10 \%$ heat-inactivated fetal bovine serum (FBS, Gibco ${ }^{\circ}$ Life Technologies) and penicillin $(100 \mathrm{U} / \mathrm{ml})$ plus streptomycin $(100 \mu \mathrm{g} / \mathrm{ml})$, and incubated at $37^{\circ} \mathrm{C}$ and $5 \% \mathrm{CO}_{2}$. Cells were seeded in a T25 flask at $5 \times 10^{5}$ cells/ml 1 day prior to inoculation.

\section{Cell proliferation assay}

RAW264.7 cells were seeded in 96-well culture plates 1 day prior to treatment at a density of $2 \times 10^{3}$ cells/well in $100 \mu \mathrm{L}$ medium, and then were treated with either phosphate buffer saline (PBS), $20 \mu \mathrm{g} / \mathrm{ml} \mathrm{rSj13,} 5 \mu \mathrm{g} / \mathrm{ml}$ concanavalin-A (ConA, positive control), or $\mathrm{rSjCa} 8$ at different concentrations ( 1 and $20 \mu \mathrm{g} / \mathrm{ml}$ ) for either 24 or 48 h. Cell viability was assessed using Cell Counting Kit-8 (CCK-8, Beyotime Biotechnology, Haimen, China) as described previously [15]. Absorbance at $450 \mathrm{~nm}$, for positive values indicate cellular proliferation, was measured using a reference wavelength of $595 \mathrm{~nm}$ with an automatic Microplate Absorbance Reader (Tecan, Austria). 


\section{Apoptosis assay}

To detect apoptosis in RAW264.7 cells after $\mathrm{rSjCa} 8$ treatment, we assessed the surface phosphatidylserine (PS) expression levels, which increase during apoptosis. Exposure of the phospholipid PS to the external leaflet of the plasma membrane occurs early in the process of apoptosis and can be measured by Annexin-V-FITC staining. Moreover, dead cells that reach late stages of apoptosis can be quantified by positive PI staining. To quantify apoptosis, an early Apoptosis Detection Kit (Beyotime Biotechnology) was performed. RAW264.7 cells $\left(10^{5}\right.$ cells/well) were grown in culture medium in the presence or absence of $\mathrm{rSjCa} 8$ ( 1 or $20 \mu \mathrm{g} / \mathrm{ml}), \mathrm{rSj} 13$ $(20 \mu \mathrm{g} / \mathrm{ml})$, or 1:2000 (V/V) Apoptosis Inducer (positive control, Beyotime Biotechnology) for 24 or $48 \mathrm{~h}$. Then, cells were harvested and pelleted by centrifugation. After washing with $\mathrm{PBS}$ and resuspension in binding buffer (10 mM HEPES/ $\mathrm{NaOH}, 140 \mathrm{mM} \mathrm{NaCl}, 2.5 \mathrm{mM} \mathrm{CaCl}_{2}$, $\mathrm{pH}$ 7.4) containing 1:100 (V/V) propidium iodide (PI) and Annexin V-FITC at room temperature for $30 \mathrm{~min}$ in the dark, cells were analyzed using either a Beckman CytoFLEX Flow Cytometer (Beckman Coulter, Brea, CA, USA), or observed using a 40× lens under an Olympus FV 500-IX81 laser-scanning confocal microscope (Olympus Microscopy, Hamburg, Germany) with an excitation wavelength of $490 \mathrm{~nm}$ and emission wavelengths of $520 \mathrm{~nm}$ for Annexin-V-FITC and $650 \mathrm{~nm}$ for PI. The apoptosis rate for each group was calculated according to the following formula: apoptosis rate $(\%)=($ number of apoptotic cells in a microscopic field/total number of cells in a microscopic field) $\times 100$.

\section{Transwell migration assay}

In vitro migration assays were performed using transwell chambers with a $10-\mu \mathrm{m}$ thin transparent polycarbonate membrane and an 8-micron polyester filter (Corning Inc. Corning, NY, USA). RAW264.7 cells $\left(7.5 \times 10^{3}\right)$ that were pretreated for $1 \mathrm{~h}$ with $20 \mu \mathrm{g} / \mathrm{ml} \mathrm{rSj} 13$ or 0,1 , or $20 \mu \mathrm{g} / \mathrm{ml} \mathrm{rSjCa} 8$ were added to the upper compartment where $1 \%$ FBS DMEM was added, whereas DMEM containing $10 \%$ FBS was added to the lower compartment. After $30 \mathrm{~h}$ incubation, the membrane between the two compartments was removed, fixed in methanol for $10 \mathrm{~min}$, and stained with Giemsa reagent. Cells on the lower surface of the filter were photographed under an Olympus BX51WI microscope (Olympus Microscopy) and counted. Five random views were photographed and quantified.

\section{Measurement of intracellular nitric oxide production}

Intracellular NO release in individual RAW264.7 cells was determined using a fluorescent dye for NO, 4amino-5-methylamino-2', $7^{\prime}$-difluorofluorescein diacetate (DAF-FM/DA, Beyotime Biotechnology) following a previously described protocol [16]. To assess the effect of $\mathrm{rSjCa} 8$ on NO production by macrophages, RAW264.7 cells were pre-stimulated with $1 \mu \mathrm{g} / \mathrm{ml}$ LPS for $24 \mathrm{~h}$ at $37{ }^{\circ} \mathrm{C}$, and then were treated for $30 \mathrm{~min}$ with various doses of $\mathrm{rSjCa} 8(0,1$, and $20 \mu \mathrm{g} / \mathrm{ml})$ or control protein $\mathrm{rSj} 13(20 \mu \mathrm{g} / \mathrm{ml})$. To analyze the dose- and timedependent inhibitory activity of $\mathrm{rSjCa} 8$ on $\mathrm{NO}$ production by macrophages, LPS-stimulated RAW264.7 cells were incubated with $20 \mu \mathrm{g} / \mathrm{ml} \mathrm{rSjCa} 8$ for $0,1,10,20$, or $30 \mathrm{~min}$. Cells from all groups were loaded with $5 \mu \mathrm{M}$ DAF-FM/DA in the dark for $30 \mathrm{~min}$ at $37{ }^{\circ} \mathrm{C}$, and then were rinsed three times. Thereafter, the fluorescence intensities of cells were measured using an Olympus FV 500-IX81 laser-scanning confocal microscope (Olympus, Microscopy) with excitation and emission wavelengths of 490 and $510 \mathrm{~nm}$, respectively. Furthermore, dynamic changes of fluorescence intensities of NO produced by LPS-stimulated macrophages after treatment with increasing concentrations of $\mathrm{rSjCa} 8(5,10,20,40,60$ and $80 \mu \mathrm{g} / \mathrm{ml}$ ) were recorded in time-series mode at $1.7 \mathrm{~s}$ intervals under a confocal microscope.

\section{Detection of intercellular $\mathrm{Ca}^{2+}$ levels}

Previous studies have shown a role for thapsigargin as an endoplasmic reticulum (ER) stressor, as well as a

Table 1 Primers used for quantitative RT- PCR

\begin{tabular}{|c|c|c|c|}
\hline Gene symbol & Forward primer & Reverse primer & Ref. \\
\hline Cat & CCTCGTTCAGGATGTGGTTT & GGCATCCCTGATGAAGAAAA & [49] \\
\hline Fas & GCAGACATGCTGTGGATCTGG & TCACAGCCAGGAGAATCGCAG & [50] \\
\hline Gadd45a & CTGCCTCCTGGTCACGAA & TTGCCTCTGCTCTCTTCACA & [51] \\
\hline Hmgbl 1 & CCATTGGTGATGTTGCAAAG & CTITITCGCTGCATCAGGTT & [52] \\
\hline Myc & GAGGCGAACACACAACGTCTT & CACGCAGGGCAAAAAAGC & [53] \\
\hline NOS2 & AACCCCTTGTGCTGTTCTCAGCC & GTGGACGGGTCGATGTCACATGC & [54] \\
\hline Sod2 & ATGTTACAACTCAGGTCGCTCTTC & TGATAGCCTCCAGCAACTCTCC & {$[55]$} \\
\hline Txnip & CAAGTTCGGCTITGAGCTTC & GCCATTGGCAAGGTAAGTGT & [56] \\
\hline 185 & GTCTGTGATGCCCTTAGA & AGCTTATGACCCGCACTTAC & [57] \\
\hline
\end{tabular}




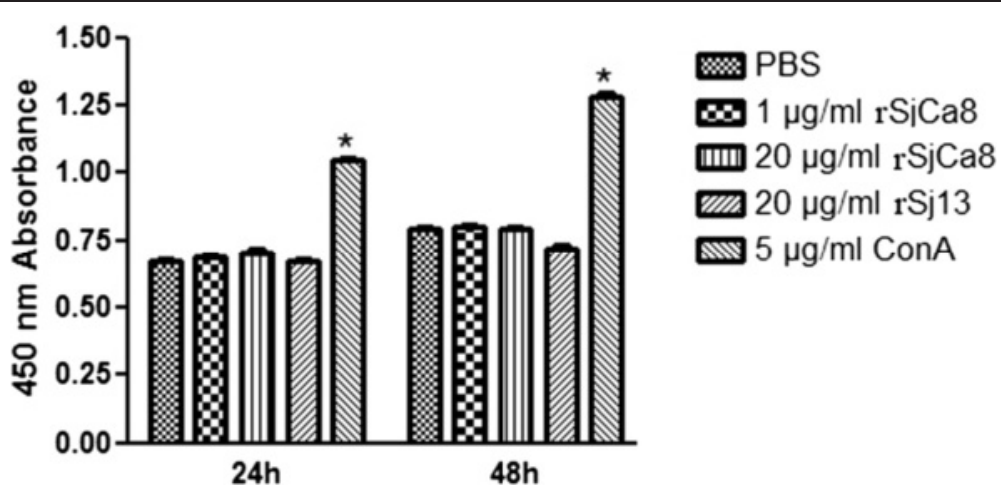

Fig. 1 Effects of rSjCa8 on the proliferation of RAW264.7 cells. RAW264.7 cells were treated or not with ConA ( $5 \mu \mathrm{g} / \mathrm{ml})$, rSj13 (20 $\mu \mathrm{g} / \mathrm{ml})$, or various concentrations of rSjCa8 (1 or $20 \mu \mathrm{g} / \mathrm{ml}$ ) for 24 or $48 \mathrm{~h}$, respectively. Cell viability was assessed in triplicate using the CCK-8 method according to the manufacturer's protocol. * $P<0.05$, compared with the PBS group

$\mathrm{Ca}^{2+}$-pump (ATPase) inhibitor that acts on the sarcoplasmic reticulum (SR) membrane [17]. In the ER stress response, apoptosis is induced by thapsigargin in a manner dependent upon $\mathrm{Ca}^{2+}$ outflow from the SR along with cytoplasmic calcium overload. EDTA functions as a chelator bound to $\mathrm{Ca}^{2+}$ to reduce intercellular calcium concentrations. To test for correlations between NO production and intracellular calcium concentrations $\left(\left[\mathrm{Ca}^{2+}\right]_{\mathrm{i}}\right)$ in macrophages, we simultaneously detected intracellular $\mathrm{NO}$ production and $\left[\mathrm{Ca}^{2+}\right]_{\mathrm{i}}$ of individual RAW264.7 cells after various treatments. Cells pretreated with LPS $(1 \mu \mathrm{g} / \mathrm{ml})$ for 24 h were divided into 6 groups: controls treated with PBS (group I), a group treated with $20 \mu \mathrm{g} / \mathrm{ml} \mathrm{rSjCa8}$ for $30 \mathrm{~min}$ (group II), an internal control group treated with $20 \mu \mathrm{g} / \mathrm{ml}$ rSj13 for $30 \mathrm{~min}$ (group III), a group treated with $20 \mu \mathrm{g} / \mathrm{ml}$ $\mathrm{rSjCa} 8+10 \mathrm{mM}$ ethylenediaminetetra-acetic acid (EDTA, a chelator of $\mathrm{Ca}^{2+}$ ) for $30 \mathrm{~min}$ (group IV), a group treated with $20 \mu \mathrm{g} / \mathrm{ml} \mathrm{rSjCa} 8+30 \mu \mathrm{M}$ thapsigargin (an inhibitor of sarco-endoplasmic reticulum $\mathrm{Ca}^{2+}$-ATPases) for $30 \mathrm{~min}$ (group V), and a group treated with $20 \mu \mathrm{g} / \mathrm{ml}$ rmuSjCa8 (group VI).

Finally, cells were loaded with $2 \mu \mathrm{M}$ Rhod-2 AM (Dojindo Kumamoto, Kumamoto, Japan; a red fluorescent probe for intercellular $\mathrm{Ca}^{2+}$ ) and $5 \mu \mathrm{M}$ DAF-FM/DA (a $\mathrm{NO}$ indicator) in the dark for $30 \mathrm{~min}$ at $37^{\circ} \mathrm{C}$. After incubation, cells were imaged using an Olympus FV 500-IX81 laser-scanning confocal microscope (Olympus Microscopy). The fluorescence of Rhod-2 AM and DAF-FM/DA were excited at $577 \mathrm{~nm}$ and $490 \mathrm{~nm}$, and emitted at $581 \mathrm{~nm}$ and $510 \mathrm{~nm}$, respectively.

\section{NO PCR microarray chip}

RAW264.7 cells were cultured, stimulated with $1 \mu \mathrm{g} / \mathrm{ml}$ LPS for $24 \mathrm{~h}$, and treated with either PBS or $\mathrm{rSjCa} 8$ $(20 \mu \mathrm{g} / \mathrm{ml})$ for $30 \mathrm{~min}$ as described above. Total RNA from cells in both groups was prepared using TRIzol ${ }^{\circ}$

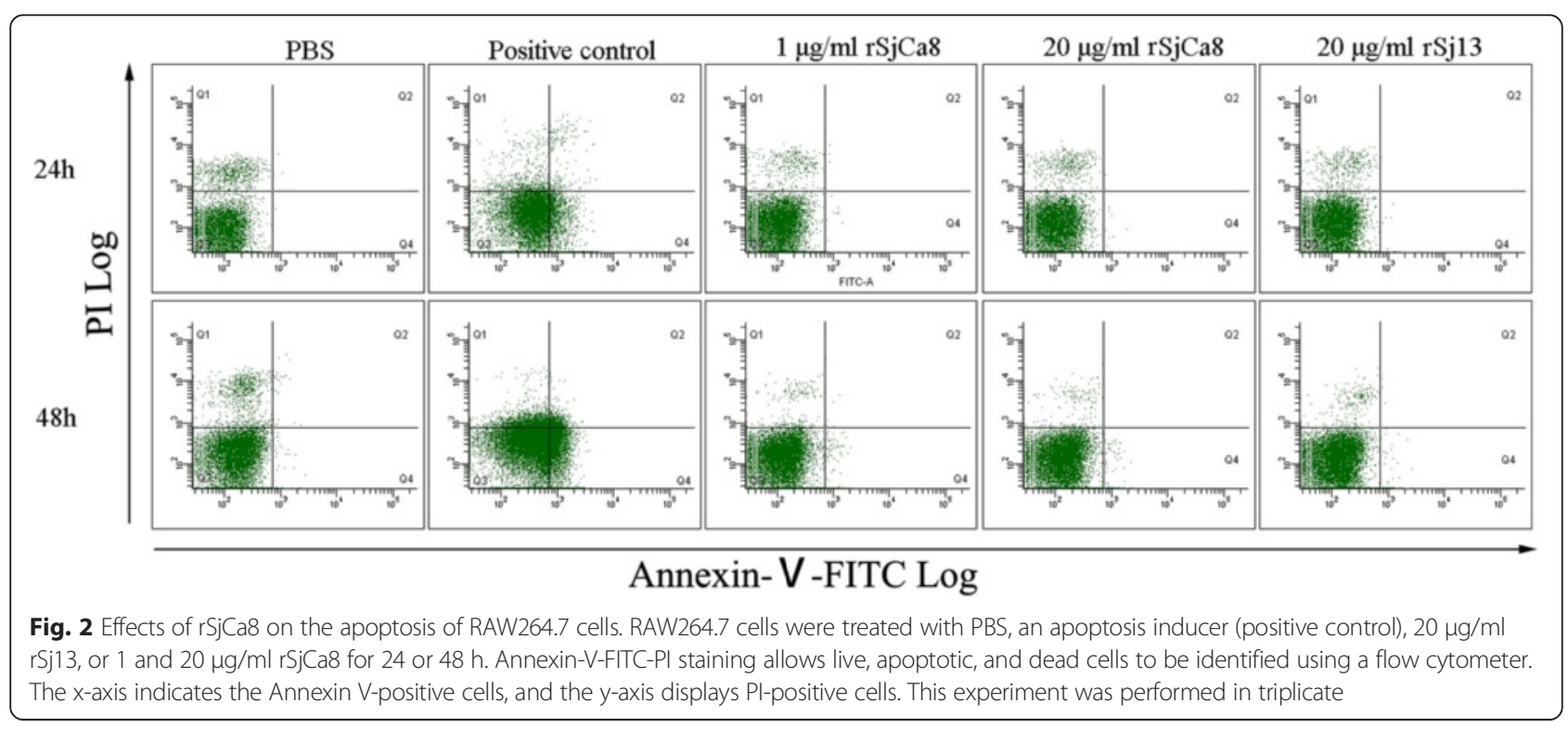


reagent (Invitrogen Life Technologies, Carlsbad, CA, USA) and the Mouse Nitric Oxide Signaling Pathway $\mathrm{RT}^{2}$ Profiler $^{\mathrm{TM}}$ PCR Array (SABiosciences/QIAGEN Company, Fredrick, MD, USA) was performed according to the manufacturer's protocol by KangChen Bio-Tech Inc. (Shanghai, China). In the statistical analyses, gene expression differences were considered significant if they showed an expression fold-change $\geq 3$ between two groups with a $p$-value $<0.05$.

\section{Real-time quantitative PCR}

A subset of genes exhibiting different expression patterns was selected for further validation using qRT-PCR.
Reactions were carried out in technical triplicates using a LightCycler ${ }^{\circ} 480$ System (Roche, Switzerland) with universal cycling conditions, as described in our previous study [13]. Specific primers for target genes and the reference gene, $18 S$ rRNA, are listed in Table 1, and the relative expression levels of each selected gene were quantified by normalization to the corresponding normalized value of the $18 S$ rRNA control (fold-change $=2^{-\Delta \Delta C T}$ ) using the software provided with the instrument.

\section{Worm recovery and tissue sampling}

To evaluate the effects of $\mathrm{rSjCa} 8$ on $\mathrm{NO}$ production and host skin penetration by $S$. japonicum larvae in vivo, a

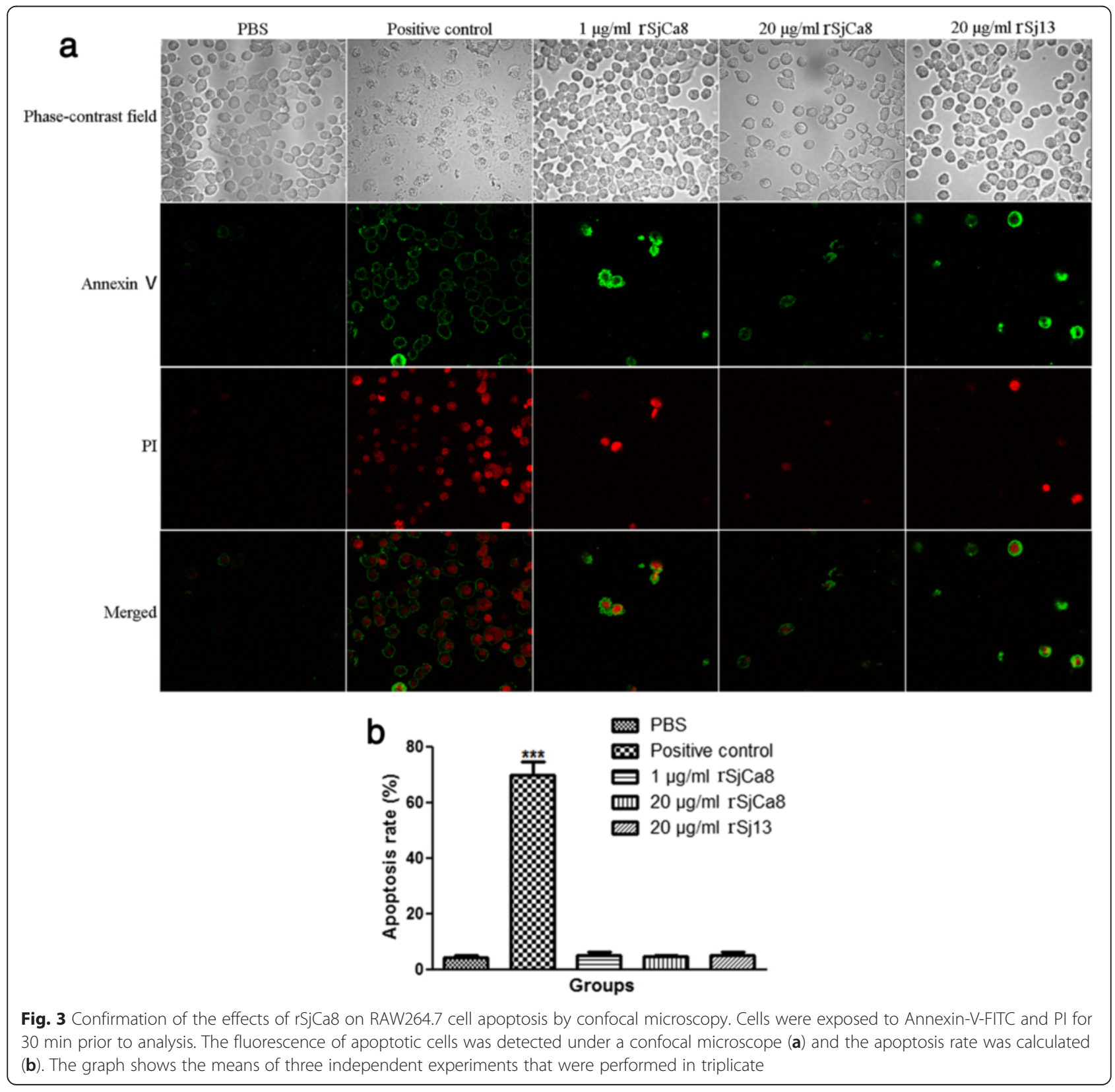




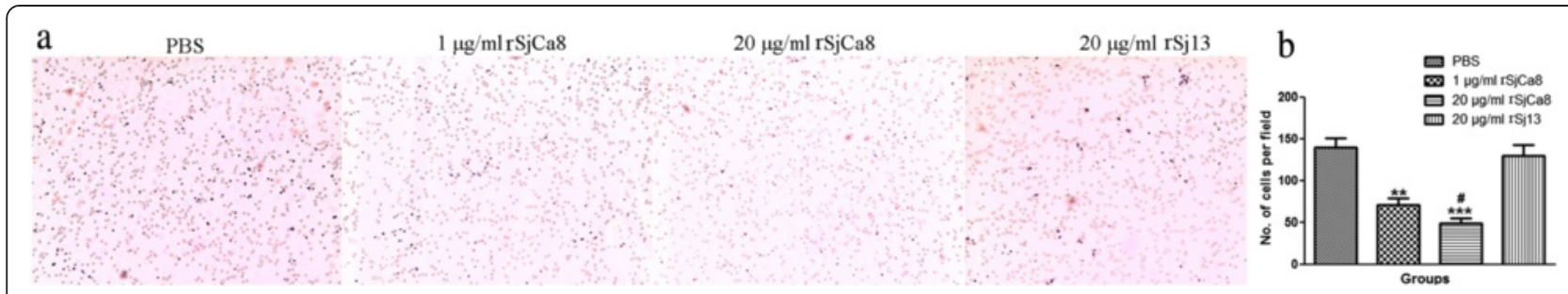

Fig. 4 Impaired migration of RAW264.7 cells caused by rSjCa8 in a transwell chamber assay. Filters were stained by Giemsa reagent (a) and the cells that passed through the filter were counted (b). The capacity of RAW264.7 cells to migrate through transwell filters was significantly inhibited by treatment of cells with $1 \mu \mathrm{g} / \mathrm{ml} \mathrm{rSjCa8}(P<0.01)$ or $20 \mu \mathrm{g} / \mathrm{ml} \mathrm{rSjCa8}(P<0.001)$, but not rSj13, compared with the PBS group. The means \pm SD of three independent experiments are shown; ${ }^{* *} P<0.01$ and ${ }^{* *} P<0.001$, compared with the PBS group; \#P<0.05, compared with the $1 \mu \mathrm{g} / \mathrm{ml}$ SjCa8 group

modified immune challenge experiment in mice was performed as described in our previous study [11]. Briefly, 40 mice were randomly divided into four groups of 10 mice each. The immune challenge group (Freund's adjuvant $+\mathrm{rSjCa} 8$ /infected group), adjuvant-treated group (Freund's adjuvant/infected group), and challenge group (infected group) were treated as described previously [11]. The immune-challenge group of mice was injected subcutaneously with $20 \mu \mathrm{g} \quad \mathrm{rSjCa} 8$ plus complete Freund's adjuvant (Sigma) and were boosted twice with the same amount of antigen with incomplete Freund's adjuvant (Sigma) at 2 -week intervals. The adjuvanttreated group of mice was subjected to the same immunization schedule as the immune-challenge group, but $\mathrm{PBS}$ replaced $\mathrm{rSjCa}$. Fourteen days after the final boost, mice in these three groups were challenged percutaneously with $50 \pm 2$ cercariae for 20 min using the cover glass method. Another group of 10 mice (uninfected group) was subjected to the same immunization schedule as the adjuvant group, but PBS was used in place of adjuvant and without a subsequent challenge infection with cercariae.

At $6 \mathrm{~h}$ post-challenge infection, all vaccinated and control mice were euthanized and the shaved skin areas that were exposed to cercariae were quickly removed. The skin tissues from half of each group of mice were cut into pieces and cultured for $24 \mathrm{~h}$ at $37{ }^{\circ} \mathrm{C}, 5 \% \mathrm{CO}_{2}$ in RPMI 1640 medium containing antibiotics $(100 \mathrm{U} / \mathrm{ml}$ penicillin and $100 \mu \mathrm{g} / \mathrm{ml}$ streptomycin) and $10 \%$ fetal calf serum (Invitrogen). In these cultured skin tissues, the effects of $\mathrm{rSjCa} 8$ on penetration and migration by cercariae were evaluated based on the number of penetrated larvae, as described previously [18]. The skin tissues from the other half of each group were cut into pieces and lysed with Lysis Buffer (Thermo Scientific/Pierce; $1 \mathrm{ml} / \mathrm{g}$ skin), and skin homogenates were prepared using a TissueLyser II (Qiagen) and centrifuged at $10,000 \mathrm{~g}$ for $5 \mathrm{~min}$ at $4{ }^{\circ} \mathrm{C}$ to collect supernatants. The Total Nitric Oxide Assay kit (Beyotime Biotechnology) was used for NO detection following the manufacturer's protocol and NO levels in the skin homogenate supernatants were determined by measuring nitrites with Griess reagent [19]. Absorbance was read at $540 \mathrm{~nm}$ with an automatic Microplate Absorbance Reader (Tecan, Austria).

\section{Statistical analysis}

Each experiment was repeated three times and data were represented as means \pm standard derivation (SD). The

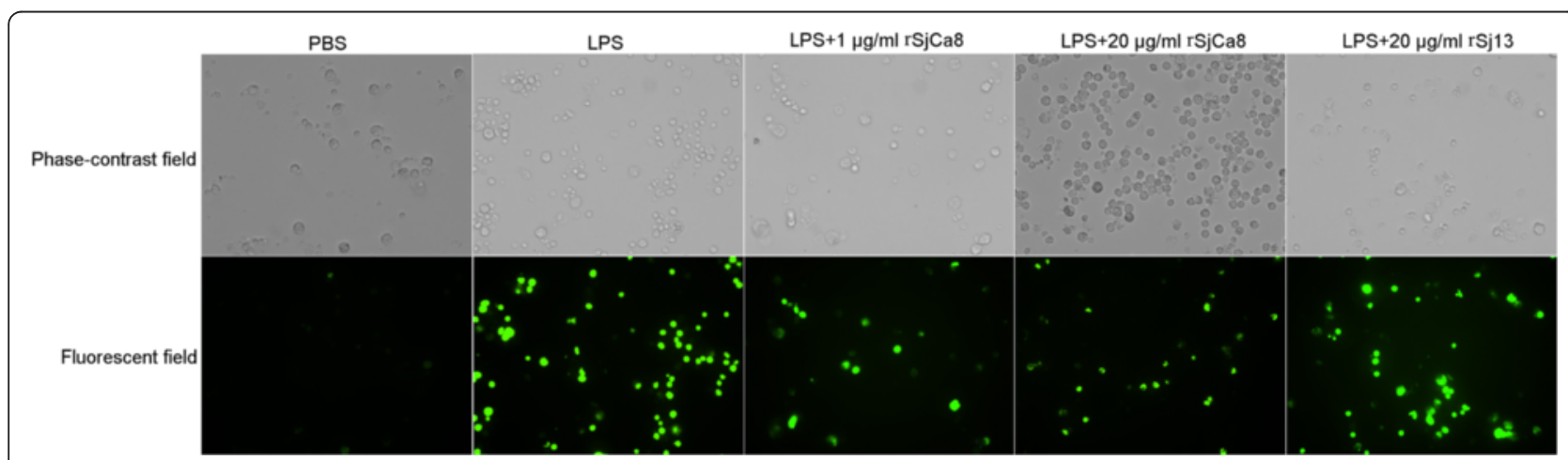

Fig. 5 Inhibition of NO release by LPS-stimulated RAW264.7 cells treated with rSjCa8. RAW264.7 cells were cultured in medium containing 1 $\mu \mathrm{g} / \mathrm{ml}$ LPS for $24 \mathrm{~h}$, and were subsequently exposed for another $30 \mathrm{~min}$ to PBS, rSjCa8 $(1$ or $20 \mu \mathrm{g} / \mathrm{ml})$, or rSj13 $(20 \mu \mathrm{g} / \mathrm{ml})$. NO generation by the cells that were loaded with the NO-specific fluorescent probe DAF-AM was detected as intracellular green fluorescence signal by laser-scanning confocal microscopy 
statistical significance of differences between groups was determined using a one-way analysis of variance (ANOVA) followed by the Tukey-Kramer test using GraphPad Prism version 5.0 (GraphPad Software Inc., La Jolla, CA, USA); $P<0.05$ was used as a threshold for statistically significant differences.

\section{Ethical Statement}

This study was carried out in strict accordance with the recommendations in the Guide for the Care and Use of Laboratory Animals of Sun Yat-sen University. The protocol was approved by the Committee on the Ethics of Animal Experiments of Zhongshan School of Medicine, Sun Yat-sen
University (Permit Number: 2010-0326). All surgery was performed under sodium pentobarbital anesthesia, and all efforts were made to minimize suffering.

\section{Results}

\section{rSjCa8 does not affect macrophage proliferation}

To assess the effects of $\mathrm{rSjCa} 8$ on the growth of RAW264.7 cells, cell viability was evaluated using the CCK-8 method. Notably, the ConA (positive control) used at test concentration significantly induced cell proliferation compared with that observed in the PBS group, whereas neither $\operatorname{rSjCa} 8(1,5$, or $20 \mu \mathrm{g} / \mathrm{ml})$ nor $\mathrm{rSj} 13$ altered the proliferation of RAW264.7 cells (Fig. 1).

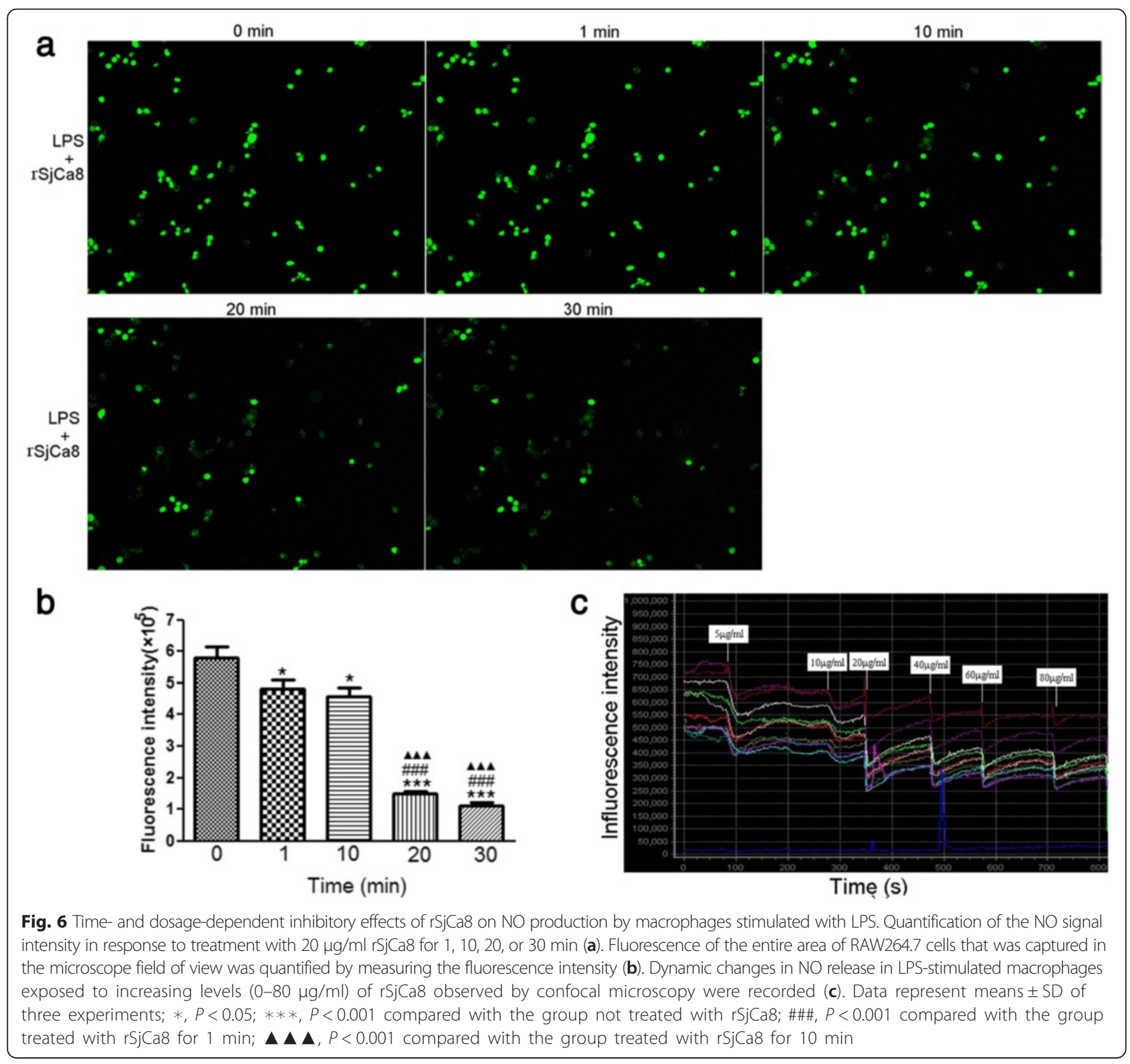




\section{rSjCa8 does not induce macrophage apoptosis}

As shown in Fig. 2, an apoptosis inducer dramatically increased the apoptosis of RAW264.7 cells compared with the PBS and $\mathrm{rSj} 13$ control group, with proportions of 35.2 and $46.1 \%$ Annexin V-positive cells after 24 and $48 \mathrm{~h}$ of treatment, respectively, whereas no obvious changes in the proportion of Annexin V-positive cells after stimulation for 24 or $48 \mathrm{~h}$ by $1 \mu \mathrm{g} / \mathrm{ml}(2.35$ and $4.16 \%$, respectively) or $20 \mu \mathrm{g} / \mathrm{ml}$ (1.98 and $3.77 \%$, respectively) $\mathrm{rSjCa} 8$ were observed (see the lower right quadrant of the dot plot), indicating a negligible effect of rSjCa8 on macrophage apoptosis.

To confirm this observation, apoptosis imaging by confocal microscopy was performed. Similar to our flow cytometry data, there was a significant 16.14-fold increase $(P<0.001)$ in apoptotic cells, which were labeled by Annexin-V-FITC and PI, in the positive control group compared with that observed in the PBS control group. However, differences between the PBS group, $1 \mu \mathrm{g} / \mathrm{ml}$ $\mathrm{rSjCa} 8$ group, $20 \mu \mathrm{g} / \mathrm{ml} \mathrm{rSjCa8}$ group, and $20 \mu \mathrm{g} / \mathrm{ml}$
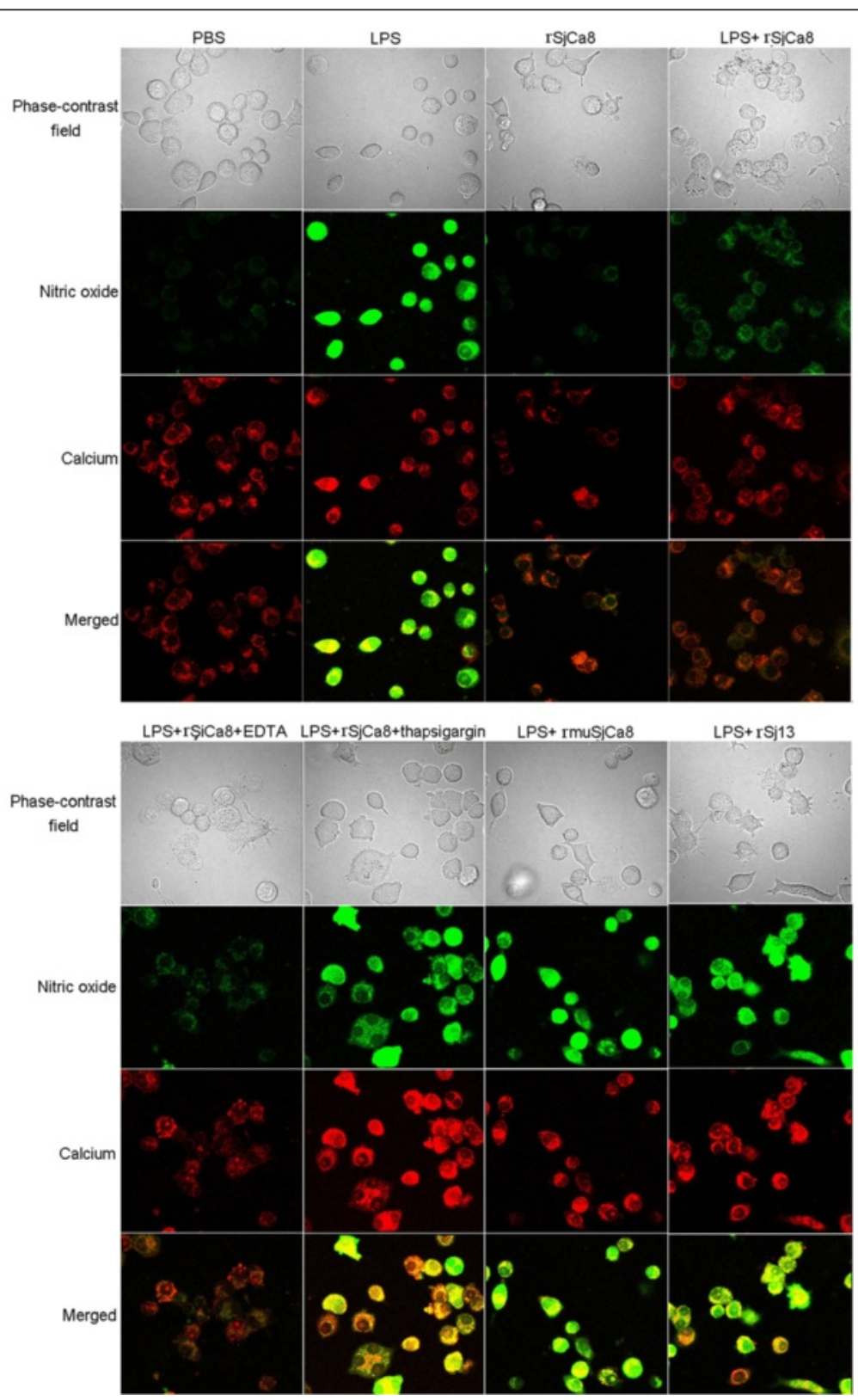

Fig. 7 Correlations between intracellular calcium levels and reduced NO generation by rSjCa8. After incubation with or without $1 \mu \mathrm{g} / \mathrm{ml}$ LPS, RAW264.7 macrophages were treated with $20 \mathrm{mg} / \mathrm{ml} \mathrm{rSjCa8}$ plus different reagents that can alter intercellular calcium level, as described in the methods section. Cells were loaded with the NO probe DAF-AM (green) and the calcium ion probe Rhod-2 AM (red). Green and red fluorescent intensities were detected simultaneously by laser-scanning capture microscopy (600x) 
rSj13 group were not significant $(P>0.05$; Fig. 3). Thus, we concluded that $\mathrm{rSjCa} 8$ treatment did not significantly affect macrophage apoptosis.

\section{rSjCa8 inhibits macrophage migration}

Down-regulation of host macrophage chemotaxis facilitates immune evasion by cercariae of $S$. japonicum when they penetrate the skin. We explored the potential effect of $\mathrm{rSjCa} 8$ on macrophage migration using a transwell system. Our results revealed that $\mathrm{rSjCa} 8$ treatment dosedependently and markedly reduced macrophage migration compared with the control group $(1 \mu \mathrm{g} / \mathrm{ml} \mathrm{rSjCa} 8$ group vs. PBS group, $P<0.01 ; 20 \mu \mathrm{g} / \mathrm{ml} \mathrm{rSjCa8}$ group vs. PBS group, $P<0.001$; Fig. 4). Therefore, $\mathrm{rSjCa} 8$ might play a vital role in limiting macrophage chemotaxis, especially when $S$. japonicum cercariae penetrate the skin of the hosts, thereby down regulating the host immune response and facilitating infection.

rSjCa8 inhibits LPS-induced NO release by RAW264.7 cells Figure 5 shows NO release in cultured LPS-stimulated RAW264.7 macrophages exposed to $\mathrm{rSjCa} 8$ and the fluorescence intensity of $\mathrm{NO}$ increased in macrophages $24 \mathrm{~h}$ after stimulation with $1 \mu \mathrm{g} / \mathrm{ml}$ LPS. Surprisingly, NO generation in RAW264.7 cells treated with LPS was significantly suppressed by $\mathrm{rSjCa} 8$ treatment in a concentration- and time-dependent manner, as indicated by the reduced intracellular fluorescent signals of the $\mathrm{NO}$ probes (Figs. 5 and 6). This finding is consistent with the changes in NO real-time fluorescence that we monitored using laser-scanning confocal microscopy (Fig. 6c), which quantitatively show diminished fluorescent intensity when the dosage of $\mathrm{rSjCa} 8$ was increased $(0-20 \mu \mathrm{g} / \mathrm{ml})$. These findings suggest that the disruption of $\mathrm{NO}$ generation in response to $\mathrm{rSjCa} 8$ occurs in macrophages.

\section{Suppression of NO release by $\mathrm{rSjCa} 8$ in RAW264.7 cells is} correlated with changes in intracellular calcium levels

To investigate correlations between cytoplasmic $\mathrm{Ca}^{2+}$ levels and the suppressive effects of $\mathrm{rSjCa} 8$ on $\mathrm{NO}$ release, either chelate (EDTA), an agonist (thapsigargin) of calcium, or a recombinant mutant $\mathrm{SjCa} 8$ protein
(rmuSjCa8) were applied to either increase or decrease levels of intracellular $\mathrm{Ca}^{2+}$ in macrophages. The data shown in Figs. 7 and 8 indicate that both cytoplasmic $\mathrm{Ca}^{2+}$ levels and NO production after LPS treatment increased compared with the PBS controls. Co-treatment with $\mathrm{rSjCa} 8$ and EDTA impaired $\mathrm{NO}$ generation and caused the elevation of $\mathrm{Ca}^{2+}$ levels, whereas NO production and intracellular $\mathrm{Ca}^{2+}$ levels were markedly enhanced in test groups treated with either thapsigargin or rmuSjCa8. Therefore, our findings indicate that NO production by macrophages stimulated with LPS may depend upon increases in cytosolic $\mathrm{Ca}^{2+}$ levels. Moreover, the suppressive effects of $\mathrm{rSjCa} 8$ on $\mathrm{NO}$ release by LPSstimulated macrophages might be a consequence of reduced intracellular $\mathrm{Ca}^{2+}$ concentrations. Moreover, Glutamate residues at positions 26 and 62 in the $\mathrm{Ca}^{2+}$-binding loops might be critical for the $\mathrm{Ca}^{2+}$ signaling inhibitory bioactivity of $\mathrm{rSjCa} 8$ that affects $\mathrm{NO}$ production.

\section{Transcriptional profiles of genes involved in the NO} signaling pathway are markedly altered in LPS-stimulated macrophages treated with $\mathrm{rSjCa} 8$

To elucidate the mechanism of $\mathrm{rSjCa} 8$-mediated inhibitory effects on NO production in LPS-stimulated macrophages, we attempted to identify genes with altered expression levels after treatment with $\mathrm{rSjCa}$. We focused on the nitric oxide pathway by quantitative real-time PCR microarray analysis to catalogue the expression of 84 genes, and identified 47 genes that were downregulated more than 3-fold, whereas 1 gene showed a greater than 3fold up-regulation (Table 2 and Additional file 1: Table S1). Notably, Myelocytomatosis oncogene (Myc, a transcription factor), Growth arrest and DNA-damage-inducible 45 alpha (Gadd45a, a gene that responds to environmental stresses), Thioredoxin interacting protein (Txnip, an oxidative stress mediator), TNF receptor superfamily member 6 (Fas, an inducer of apoptosis), Superoxide dismutase 2 (Sod2, an anti-oxidant gene), High mobility group box 1 (Hmgb1, a cytokine mediator of inflammation), and Nitric oxide synthase 2 (Nos2, an enzyme that catalyzes the production of $\mathrm{NO})$ were down-regulated by 1868.05-, 367.26-, 325.89-, 279.80-, 232.28-, 41.98-, and 15.64-folds,
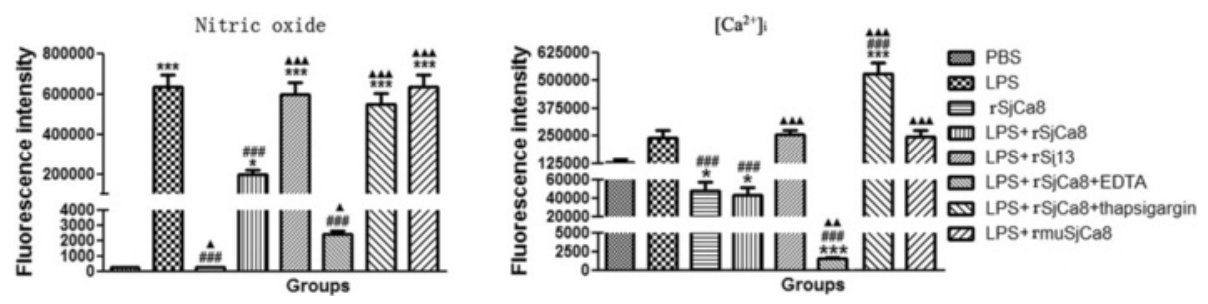

Fig. 8 Quantification of $\mathrm{NO}$ and calcium fluorescence in macrophages exposed to various in vitro stimuli. Significant differences between means are indicated; ${ }^{*} P<0.05,{ }^{* *} P<0.001$ compared with untreated cells; \#\#\#P<0.001 compared with LPS-stimulated cells; $\boldsymbol{\Delta}<0.05, \boldsymbol{\Delta} \boldsymbol{\Delta} P<0.01$, $\boldsymbol{\Delta} \boldsymbol{\Delta} \boldsymbol{\Delta} P<0.001$ compared with LPS-stimulated cells exposed to rSjCa8 
Table 2 General information of differentially expressed genes from LPS + rSjCa8 treated macrophages compared to LPS treated macrophages

\begin{tabular}{|c|c|c|c|c|c|}
\hline Well & RefSeq & Symbol & Description & $\begin{array}{l}\text { T-test } \\
P \text { value }\end{array}$ & $\begin{array}{l}\text { Fold Difference } \\
(\text { LPS + rSjCa8)/LPS }\end{array}$ \\
\hline$\overline{\mathrm{A} 02}$ & NM_028717 & Als2 & Amyotrophic lateral sclerosis 2 (juvenile) homolog (human) & $3.38 \mathrm{E}-04$ & -4.14 \\
\hline $\mathrm{A} 04$ & NM_007527 & Bax & Bcl2-associated $X$ protein & $6.11 \mathrm{E}-08$ & 10.79 \\
\hline A05 & NM_009743 & $\mathrm{BCl} 2 \mathrm{|l}$ & Bcl2-like 1 & $4.58 \mathrm{E}-06$ & -3.43 \\
\hline A08 & NM_009795 & Capns1 & Calpain, small subunit 1 & $1.58 \mathrm{E}-03$ & -3.57 \\
\hline A09 & NM_009804 & Cat & Catalase & 4.22E-07 & 1012.32 \\
\hline B01 & NM_016892 & Ccs & Copper chaperone for superoxide dismutase & $2.32 \mathrm{E}-08$ & -13.69 \\
\hline B03 & NM_007669 & Cdkn1a & Cyclin-dependent kinase inhibitor 1A (P21) & $1.47 \mathrm{E}-04$ & -7.75 \\
\hline B05 & NM_007806 & Cyba & Cytochrome b-245, alpha polypeptide & 2.05E-04 & -7.35 \\
\hline B08 & NM_007864 & Dlg4 & Discs, large homolog 4 (Drosophila) & 8.65E-05 & -9.50 \\
\hline $\mathrm{C} 01$ & NM_007987 & Fas & Fas (TNF receptor superfamily member 6) & 1.30E-04 & -279.80 \\
\hline $\mathrm{CO} 2$ & NM_010234 & Fos & FBJ osteosarcoma oncogene & $3.73 \mathrm{E}-05$ & -14.66 \\
\hline $\mathrm{CO} 4$ & NM_007836 & Gadd45a & Growth arrest and DNA-damage-inducible 45 alpha & 2.80E-08 & -367.26 \\
\hline $\mathrm{C} 05$ & NM_008160 & Gpx1 & Glutathione peroxidase 1 & $1.12 \mathrm{E}-04$ & -5.45 \\
\hline $\mathrm{CO}$ & NM_008161 & Gpx3 & Glutathione peroxidase 3 & $2.58 \mathrm{E}-05$ & -23.15 \\
\hline D01 & NM_010439 & Hmgb1 & High mobility group box 1 & $2.78 \mathrm{E}-07$ & -41.98 \\
\hline D02 & NM_008281 & Hpn & Hepsin & 4.79E-04 & -3.87 \\
\hline D03 & NM_010497 & ldh1 & Isocitrate dehydrogenase $1(\mathrm{NADP}+)$, soluble & $3.20 \mathrm{E}-06$ & -8.61 \\
\hline D04 & NM_008326 & $\operatorname{lrgm} 1$ & Immunity-related GTPase family M member 1 & $2.26 \mathrm{E}-04$ & -8.25 \\
\hline D05 & NM_010786 & Mdm2 & Transformed mouse 3 T3 cell double minute 2 & $1.09 \mathrm{E}-05$ & -14.94 \\
\hline D07 & NM_010849 & Myc & Myelocytomatosis oncogene & $1.18 \mathrm{E}-09$ & -1868.05 \\
\hline D08 & NM_010877 & Ncf2 & Neutrophil cytosolic factor 2 & $1.32 \mathrm{E}-04$ & -3.86 \\
\hline D09 & NM_008682 & Nedd1 & Neural precursor cell expressed, developmentally down-regulated gene 1 & $2.38 \mathrm{E}-06$ & -18.38 \\
\hline D12 & NM_010927 & Nos2 & Nitric oxide synthase 2 , inducible & $1.46 \mathrm{E}-06$ & -15.64 \\
\hline E02 & NM_172203 & Nox1 & NADPH oxidase 1 & $1.46 \mathrm{E}-06$ & -3.30 \\
\hline E07 & NM_011063 & Pea15a & Phosphoprotein enriched in astrocytes $15 \mathrm{~A}$ & $3.44 \mathrm{E}-05$ & -11.22 \\
\hline E08 & NM_133819 & Ppp1r15b & Protein phosphatase 1 , regulatory (inhibitor) subunit $15 \mathrm{~b}$ & 5.35E-08 & -7.67 \\
\hline E09 & NM_008913 & Рpp3ca & Protein phosphatase 3 , catalytic subunit, alpha isoform & 3.61E-07 & -24.03 \\
\hline E10 & NM_011034 & $\operatorname{Prd} \times 1$ & Peroxiredoxin 1 & $3.28 \mathrm{E}-04$ & -4.18 \\
\hline E11 & NM_011563 & $\operatorname{Prd} \times 2$ & Peroxiredoxin 2 & $1.30 \mathrm{E}-06$ & -12.04 \\
\hline E12 & NM_007453 & Prdx6 & Peroxiredoxin 6 & $3.91 \mathrm{E}-04$ & -3.19 \\
\hline F02 & NM_011170 & Prnp & Prion protein & 5.97E-05 & -12.71 \\
\hline F06 & NM_009029 & $\mathrm{Rb} 1$ & Retinoblastoma 1 & $2.40 \mathrm{E}-05$ & -16.42 \\
\hline F07 & NM_058214 & Recql4 & RecQ protein-like 4 & $4.72 \mathrm{E}-07$ & -11.70 \\
\hline F09 & NM_009101 & Rras & Harvey rat sarcoma oncogene, subgroup $R$ & $4.22 \mathrm{E}-07$ & -16.76 \\
\hline F10 & NM_009127 & Scd 1 & Stearoyl-Coenzyme A desaturase 1 & $1.01 \mathrm{E}-07$ & -35.25 \\
\hline F11 & NM_009128 & $\operatorname{Scd} 2$ & Stearoyl-Coenzyme A desaturase 2 & 7.52E-05 & -6.71 \\
\hline $\mathrm{F} 12$ & NM_024450 & $\operatorname{scd} 3$ & Stearoyl-coenzyme A desaturase 3 & $3.54 \mathrm{E}-04$ & -7.59 \\
\hline G02 & NM_011434 & Sod1 & Superoxide dismutase 1 , soluble & $1.78 \mathrm{E}-03$ & -4.30 \\
\hline G03 & NM_013671 & Sod2 & Superoxide dismutase 2, mitochondrial & 5.07E-08 & -232.28 \\
\hline G06 & NM_011640 & Trp53 & Transformation related protein 53 & 1.09E-04 & -9.62 \\
\hline G07 & NM_023719 & Txnip & Thioredoxin interacting protein & $7.36 \mathrm{E}-09$ & -325.89 \\
\hline G08 & NM_013711 & Txnrd2 & Thioredoxin reductase 2 & $6.01 \mathrm{E}-08$ & -37.67 \\
\hline
\end{tabular}


Table 2 General information of differentially expressed genes from LPS + rSjCa8 treated macrophages compared to LPS treated macrophages (Continued)

\begin{tabular}{llllll}
\hline G09 & NM_019639 & Ubc & Ubiquitin C & $1.28 \mathrm{E}-04$ & -4.20 \\
G11 & NM_026119 & Med4 & Mediator of RNA polymerase II transcription, subunit 4 homolog (yeast) & $2.78 \mathrm{E}-06$ & -3.39 \\
H01 & NM_010368 & Gusb & Glucuronidase, beta & $4.77 \mathrm{E}-02$ & -5.90 \\
H02 & NM_013556 & Hprt1 & Hypoxanthine guanine phosphoribosyl transferase 1 & $1.60 \mathrm{E}-05$ & -23.66 \\
H04 & NM_008084 & Gapdh & Glyceraldehyde-3-phosphate dehydrogenase & $1.60 \mathrm{E}-04$ & -6.13 \\
H05 & NM_007393 & Actb & Actin, beta & $3.51 \mathrm{E}-03$ & -8.43 \\
\hline
\end{tabular}

- genes that were downregulated

respectively, whereas the expression of the anti-oxidative stress gene catalase (Cat) was up-regulated by 1012.32fold (Table 2).

\section{Validation of differentially expressed genes detected by microarray analyses}

Changes in the expression levels of the most significantly differentially expressed genes identified by the microarray analyses were validated using quantitative RT-PCR to measure individual transcript levels. As shown in Fig. 9, our RT-PCR data confirmed the significant downregulation of Myc (314.47-fold reduction), Gadd45a (58.14-fold reduction), Txnip (33.50-fold reduction), Fas (173.91-fold reduction), Sod2 (34.22-fold reduction), Hmgb1 (8.76-fold reduction), and Nos2 (5.01-fold reduction), and the upregulation of Cat (534.67-fold increase) in the LPS-stimulated macrophages after exposure to $20 \mu \mathrm{g} / \mathrm{ml} \mathrm{rSjCa} 8$ for $30 \mathrm{~min}$.

Vaccination of rSjCa8 increase NO concentration in the challenging skin area and reduce the number of larvae that penetrate and migrate through the skin

The inhibitory efficacy was tested by quantifying the number of skin-stage schistosomula and effects on $\mathrm{NO}$ release that were induced by vaccination with $\mathrm{rSjCa} 8$ following challenge with $50 \pm 2$ cercariae. Our data showed that immunization with adjuvant $+\mathrm{rSjCa} 8$ resulted in a significant $(P<0.001)$ reduction by $49.39 \%$ in the number of larvae that we recovered compared with that recovered from control mice (Fig. 10b). By contrast, NO release in skin homogenates was significantly increased in infected animals after vaccination with adjuvant + $\mathrm{rSjCa} 8$ compared with that detected in uninfected mice, infected mice without vaccination, or infected mice with adjuvant vaccination alone $(P<0.01$; Fig. $10 \mathrm{c})$. The numbers of recovered schistosomula from infected mice versus infected mice treated with adjuvant, and the NO levels among uninfected mice, infected mice without vaccination, and infected mice with adjuvant vaccination alone were not significantly different (Fig. 10b, c).

\section{Discussion}

$\mathrm{Ca}^{2+}$ and $\mathrm{NO}$ are two important cellular messengers, and $\mathrm{Ca}^{2+}$ fluxes have been recognized to account for NO production [20]. Researchers have confirmed that both $\mathrm{Ca}^{2+}$ fluxes and calcium-related proteins, such as regucalcin, calmodulin, and CBPs, are thought to affect NO synthesis and release by a range of somatic cells, including renal cortical cells, neurons, macrophages, and plant cells [21-25]. In the innate immune response, calciumbinding proteins act as potential sensors that are activated by pathogen-induced calcium influx. Increased cytoplasmic $\mathrm{Ca}^{2+}$ levels trigger the activation of various downstream protein targets that affect numerous signal transduction cascades, including the NOS (nitric oxide synthase) pathway that has been associated with increased NO generation after pathogen infection in vivo and in vitro [26]. However, the importance of calciumbinding proteins from pathogens (e.g., parasites, bacteria, or viruses) on macrophage release or functions in the host has not yet been directly established [27-29]. To date, $\mathrm{SjCa} 8$ is the only CBP known to be stage-specifically expressed in schistosomal cercaria and skin-stage schistosomula [11]. Our findings originally indicated that $\mathrm{rSjCa} 8$ could significantly inhibit macrophage migration and NO release, despite its minimal impact on macrophage proliferation and apoptosis. Furthermore, our present findings show that mutations in the EF-hand motif of $\mathrm{rSjCa} 8$ or administration of thapsigargin (an agonist of storeoperated $\mathrm{Ca}^{2+}$ channels) can reverse $\mathrm{rSjCa}$ 8-induced inhibitory effects on NO release in LPS-stimulated macrophages demonstrated that the inhibition effects might be regulated by $\mathrm{Ca}^{2+}$-dependent signaling. We have now made the exciting observation that $\mathrm{rSjCa} 8$ can both modulate intracellular $\mathrm{Ca}^{2+}$ levels and also inhibit macrophage migration and NO production in the cercariaechallenged host, thereby suppressing host cell-mediated killing or elimination effects on a pathogen.

Recent work indicates that LPS, a ubiquitous component of gram-negative bacteria, can elicit innate immune responses in both animals and plants by functioning as a pathogen-associated molecular pattern (PAMP)like molecule, which can evoke $\mathrm{NO}$ generation in 

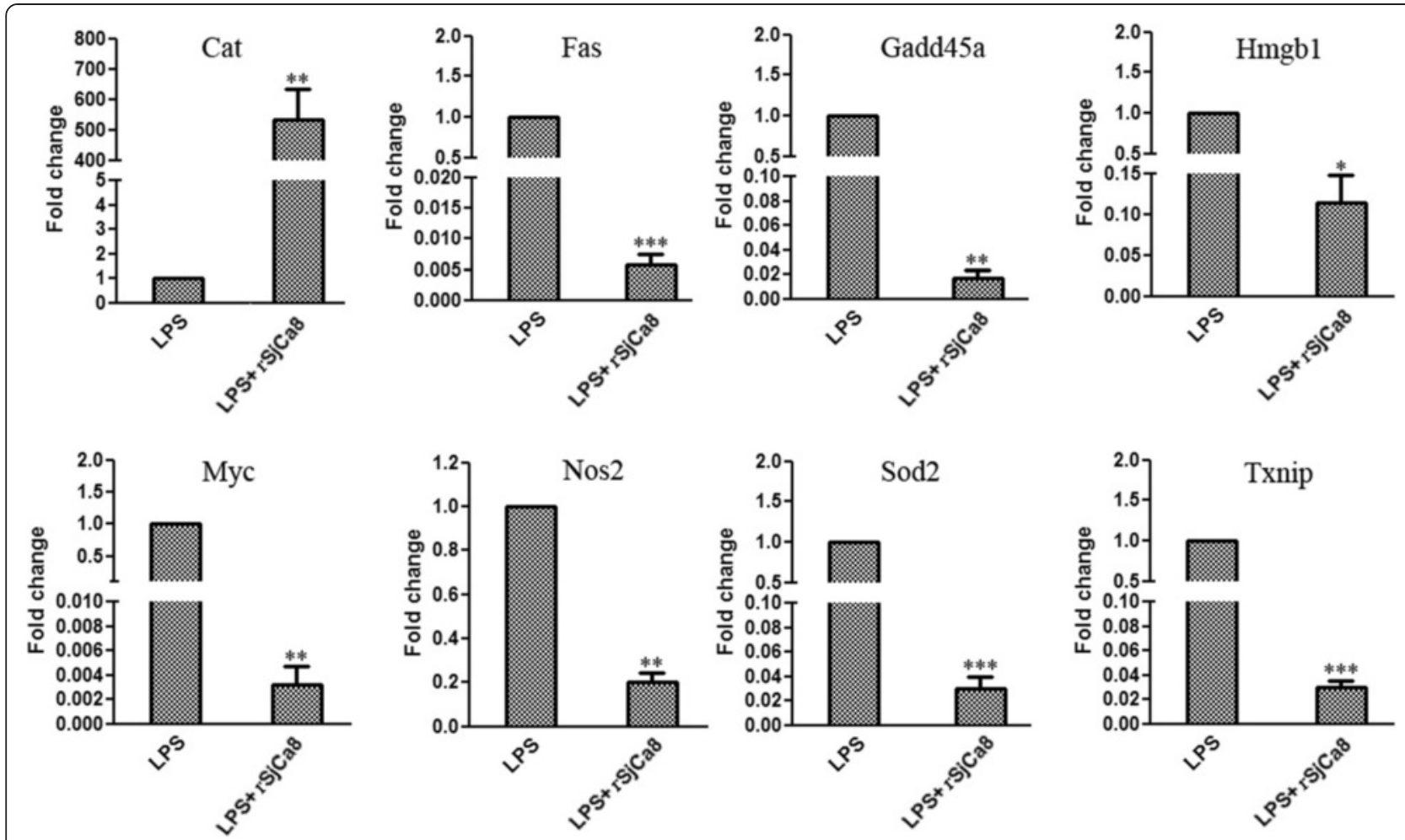

Fig. 9 Measurements of mRNA levels of the most differentially expressed genes observed in microarrays. The eight most differentially expressed genes (Cat, Fas, Gadd45a, Hmgb1, Myc, Nos2, Sod2, and Txnip) were selected to determine mRNA expression levels by quantitative real-time PCR using specific primers. Values shown represent means $\pm S D ; *, P<0.05 ; * *, P<0.01$; and $* * *, P<0.001$ compared with the LPS-stimulated group

macrophages [30], demonstrating a useful model of RAW264.7 macrophages upon LPS stimulation for investigating pathogen-mediated $\mathrm{NO}$ signaling cascades. Based on our microarray NO chip analysis and quantitative real-time PCR results, we can conclude that the mechanisms of inhibiting $\mathrm{NO}$ release by $\mathrm{rSjCa} 8$ may include up-regulated expression of Catalase-encoding gene (Cat) and down-regulated expression of 47 other genes that include Myelocytomatosis oncogene (Myc), Superoxide dismutase 2 (Sod2), Thioredoxin interacting protein
(Txnip), Growth arrest and DNA-damage- inducible 45 alpha (Gadd45a), TNF receptor superfamily member 6 (Fas), High mobility group box 1 (Hmgb1), and Nitric oxide synthase 2 (Nos2), which have been established to be involved in NO production and biological functions [31-40]. Since LPS-stimulated iNOS activation in RAW 264.7 macrophages is reactive oxygen species (ROS)dependent [41-43], the observation that extremely high expression level of Cat (an important molecule in protecting the cell from oxidative damage by ROS) in LPS-

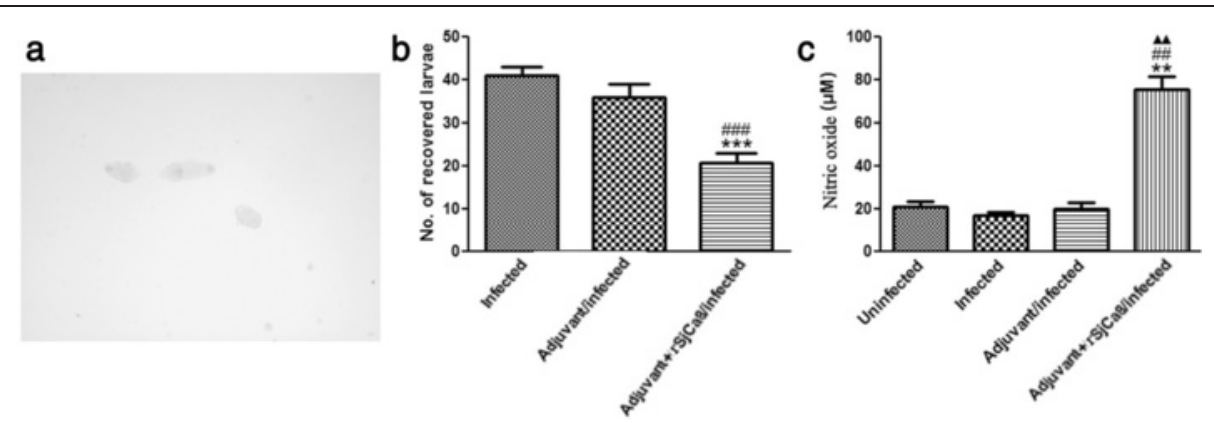

Fig. 10 Effects of vaccination of $\mathrm{rSjCa8}$ on NO production in the skin and on the number of invaded larvae. Photomicrographs ( $\times 40)$ of recovered schistosomula from different experimental groups as observed using a microscope (a). Harvested larvae of S. japonicum were counted (b), and NO concentrations in the skin homogenates were determined by absorbance at a 540-nm wavelength using a total nitric oxide assay kit; ${ }^{* *} P<0.01,{ }^{* *} P<0.001$ compared with uninfected mice; \#\# $P<0.01$, \#\#\# $<0.001$ compared with the adjuvant/infected group; $\boldsymbol{\Delta} \boldsymbol{\Delta} P<0.01$ compared with the adjuvant + rSjCa8/infected group. Experiments were replicated three times 
pretreated macrophages is induced by $\mathrm{rSjCa} 8$ suggests $\mathrm{ROS}$ are involved in the inhibitory effect of $\mathrm{rSjCa} 8$ on NO release. LPS-stimulated macrophages are a critical component of innate immunity. After LPS treatment, protein-tyrosine kinase mediates phospholipase $\mathrm{C}$ phosphorylation, which is followed by increased intracellular calcium concentrations. The increases in calcium levels sequentially trigger the activation of downstream signaling pathways that lead to inducible nitric-oxide synthase $[44,45]$. Our findings presented herein clearly show that although $\mathrm{rSjCa} 8$ clearly inhibits Toll-like receptor (TLR) activation-induced (e.g., LPS exposure) NO production, non-TLR agonists (e.g., thapsigargin exposure) can abolish the reduction in $\mathrm{NO}$ and intracellular $\mathrm{Ca}^{2+}$ accumulation in LPS-stimulated macrophages. Thus, inhibition of $\mathrm{Ca}^{2+}$-mediated inflammatory pathways likely represents a novel schistosome-induced immunosuppressive mechanism. Deeper insights into the molecules and mechanisms related to the induction of NO production by $\mathrm{rSjCa} 8$ both permit a more thorough characterization of the NO signaling system and lead to a better understanding of immune evasion by S. japonicum.

Increasing studies have confirmed the existence of cross-talk between $\mathrm{Ca}^{2+}$ signaling and $\mathrm{NO}$ in response to pathogen invasion [46-48]. To further test our hypothesis that immune evasion of cercariae during skin penetration was mediated by the inhibitory activity of $\mathrm{SjCa} 8$ on NO production, we immunized mice with adjuvant $+\mathrm{rSjCa} 8$ to induce significantly higher levels of specific anti-rSjCa8 antibodies compared to those in mice immunized with adjuvant or PBS alone (data not shown). Our findings indicate that the inhibitory activity of $\mathrm{rSjCa} 8$ on $\mathrm{NO}$ production can be suppressed by neutralizing antibodies to $\mathrm{rSjCa} 8$. Therefore, accumulation of $\mathrm{NO}$ in the challenging skin area of infected hosts caused the partial elimination of invading larvae, which can further illustrate why schistosomal cercariae are scarcely killed or eliminated while penetrating into the host's skin. Cercariae and skin-stage schistosomula might evade the host immune system-mediated elimination by secreting $\mathrm{SjCa} 8$, a stage-specific $\mathrm{CBP}$, which can inhibit macrophage migration and NO release. Therefore, $\mathrm{SjCa} 8$ is thought to be a potential chemotherapeutic target or/and vaccine candidate against schistosome infection.

\section{Conclusion}

Our findings support the conclusion that $\mathrm{SjCa} 8$, a cercaria and skin-stage schistosomulum specific CBP, inhibits cell migration and $\mathrm{Ca}^{2+}$-dependently suppresses NO release by LPS-stimulated RAW264.7 macrophages. It is feasible that, via this mechanism, $\mathrm{SjCa} 8$ can contribute to preventing larvae from the damaging or killing effects of macrophages, thus suggesting a further protective effect of this immunoregulatory molecule against host immune attack to promote the invasion, migration, and survival of cercariae. Our findings provide molecular insights that could be harnessed to develop a novel effective vaccine or drug against cercariae invasion for the prevention and control of schisotosomiasis.

\section{Additional file}

\section{Additional file 1: Microarray Analysis of 84 Genes Involved in NO} Signaling. (XLSX $20 \mathrm{~kb}$ )

\section{Competing interests}

The authors declare that they have no competing interests.

\section{Authors' contributions}

LZY conceived and designed the experiments; L, PT, YX, XYY, LJY, SX and ZHQ performed the experiments; OK, YL, and WZD analyzed data; LZY wrote the manuscript; all authors read and approved the final version of the text.

\section{Acknowledgements}

We thank Mrs. Guan Yuanjun and Mrs. Jie Liu for providing help with confocal microscopy and expert technical assistance. This work was supported by grants from the National Natural Science Foundation of China (grant no. 30800966, 81572014, 81371836 and 81572023), the Laboratory of Parasite and Vector Biology, MOPH (grant no. WSBKTKT201401), the Guangdong Natural Science Foundation (grant no. 2014A030313134), the 111 Project (grant no. B12003), the Cultivation Foundation of the Young Teacher of Sun Yat-sen University (grant no. 09ykpy77), and the Research Foundation for Students of Sun Yat-sen University (2012 and 2014).

\section{Author details}

${ }^{1}$ Zhongshan School of Medicine, Sun Yat-sen University, 74 2nd Zhongshan Road, Guangzhou 510080, China. ${ }^{2}$ Key Laboratory for Tropical Diseases Control of Ministry of Education, Sun Yat-sen University, Guangzhou 510080 China. ${ }^{3}$ Department of Social and Environmental Medicine, Faculty of Tropical Medicine, Mahidol University, Bangkok 10400, Thailand.

Received: 2 September 2015 Accepted: 28 September 2015 Published online: 07 October 2015

\section{References}

1. Hotez PJ, Kamath A. Neglected tropical diseases in sub-saharan Africa: review of their prevalence, distribution, and disease burden. PLoS Negl Trop Dis. 2009;3:e412

2. Elmorshedy H, Bergquist R, El-Ela NE, Eassa SM, Elsakka EE, Barakat R. Can human schistosomiasis mansoni control be sustained in high-risk transmission foci in Egypt? Parasit Vectors. 2015;8:372.

3. Xu X, Sun J, Zhang J, Wellems D, Qing X, McCutchan T, et al. Having a pair: the key to immune evasion for the diploid pathogen Schistosoma japonicum. Sci Rep. 2012;2:346.

4. Fishelson Z. Novel mechanisms of immune evasion by Schistosoma mansoni. Mem Inst Oswaldo Cruz. 1995;90:289-92.

5. Doenhoff MJ, Cioli D, Utzinger J. Praziquantel: mechanisms of action, resistance and new derivatives for schistosomiasis. Curr Opin Infect Dis. 2008;21:659-67

6. Lin D, Tian F, Wu H, Gao Y, Wu J, Zhang D, et al. Multiple vaccinations with UV- attenuated cercariae in pig enhance protective immunity against Schistosoma japonicum infection as compared to single vaccination. Parasit Vectors. 2011;4:103.

7. Tian F, Lin D, Wu J, Gao Y, Zhang D, Ji M, et al. Immune events associated with high level protection against Schistosoma japonicum infection in pigs immunized with UV-attenuated cercariae. PLoS One. 2010;5:e13408.

8. Oswald IP, Eltoum I, Wynn TA, Schwartz B, Caspar P, Paulin D, et al. Endothelial cells are activated by cytokine treatment to kill an intravascular 
parasite, Schistosoma mansoni, through the production of nitric oxide. Proc Natl Acad Sci U S A. 1994;91:999-1003.

9. Wynn TA, Oswald IP, Eltoum IA, Caspar P, Lowenstein CJ, Lewis FA, et al. Elevated expression of Th1 cytokines and nitric oxide synthase in the lungs of vaccinated mice after challenge infection with Schistosoma mansoni. J Immunol. 1994;153:5200-9.

10. Knudsen GM, Medzihradszky KF, Lim KC, Hansell E, McKerrow JH. Proteomic analysis of Schistosoma mansoni cercarial secretions. Mol Cell Proteomics. 2005;4:1862-75.

11. Lv ZY, Yang LL, Hu SM, Sun $X$, He HJ, He SJ, et al. Expression profile, localization of an 8-kDa calcium-binding protein from Schistosoma japonicum (SjCa8), and vaccine potential of recombinant SjCa8 (rSjCa8) against infections in mice. Parasitol Res. 2009;104:733-43.

12. Zhou YP, Wu ZD, Yang LL, Sun X, You X, Yu XB, et al. Cloning, molecular characterization of a 13-kDa antigen from Schistosoma japonicum, Sj13, a putative salivary diagnosis candidate for Schistosomiasis japonica. Parasitol Res. 2009;105:1435-44.

13. Ji P, Hu H, Yang X, Wei $X$, Zhu C, Liu J, et al. AcCystatin, an immunoregulatory molecule from Angiostrongylus cantonensis, ameliorates the asthmatic response in an aluminium hydroxide/ovalbumin- induced rat model of asthma. Parasitol Res. 2015;114:613-24

14. Maune JF, Klee CB, Beckingham K. Ca ${ }^{2+}$ binding and conformational change in two series of point mutations to the individual $\mathrm{Ca}(2+)$-binding sites of calmodulin. J Biol Chem. 1992;267:5286-95.

15. Wu ZS, Wu Q, Wang CQ, Wang XN, Huang J, Zhao JJ, et al. miR-340 inhibition of breast cancer cell migration and invasion through targeting of oncoprotein c-Met. Cancer. 2011;117:2842-52.

16. Asai M, Takeuchi $K$, Saotome M, Urushida T, Katoh H, Satoh H, et al. Extracellular acidosis suppresses endothelial function by inhibiting store-operated $\mathrm{Ca}^{2+}$ entry via non-selective cation channels. Cardiovasc Res. 2009:83:97-105.

17. Bachar E, Ariav Y, Ketzinel-Gilad M, Cerasi E, Kaiser N, Leibowitz G. Glucose amplifies fatty acid-induced endoplasmic reticulum stress in pancreatic beta-cells via activation of mTORC1. PLOS One. 2009;4:e4954.

18. Yang F, Long E, Wen J, Cao L, Zhu C, Hu H, et al. Linalool, derived from Cinnamomum camphora (L.) Presl leaf extracts, possesses molluscicidal activity against Oncomelania hupensis and inhibits infection of Schistosoma japonicum. Parasit Vectors. 2014;7:407.

19. Zhang H, Li W, Wang G, Su Y, Zhang C, Chen X, et al. The distinct binding properties between avian/human influenza A virus NS1 and Postsynaptic density protein-95 (PSD-95), and inhibition of nitric oxide production. Virol J. 2011;8:298.

20. López-Jaramillo P. Calcium, nitric oxide, and preeclampsia. Semin Perinatol. 2000:24:33-6.

21. Ma ZJ, Yamaguchi M. Regulatory effect of regucalcin on nitric oxide synthase activity in rat kidney cortex cytosol: Role of endogenous regucalcin in transgenic rats. Int J Mol Med. 2003;12:201-6.

22. Luo CX, Zhu DY. Research progress on neurobiology of neuronal nitric oxide synthase. Neurosci Bull. 2011;27:23-35.

23. Zhang B, Crankshaw W, Nesemeier R, Patel J, Nweze I, Lakshmanan J, et al. Calcium-mediated signaling and calmodulin-dependent kinase regulate hepatocyte-inducible nitric oxide synthase expression. J Surg Res. 2015;193:795-801.

24. Lecourieux D, Ranjeva R, Pugin A. Calcium in plant defence-signalling pathways. New Phytol. 2006;171:249-69.

25. Müller F, Koch KW. Calcium-binding proteins and nitric oxide in retinal function and disease. Acta Anat (Basel). 1998;162:142-50.

26. Engels FH, Koski GK, Bedrosian I, Xu S, Luger S, Nowell PC, et al. Calcium signaling induces acquisition of dendritic cell characteristics in chronic myelogenous leukem ia myeloid progenitor cell. Proc Natl Acad Sci U S A. 1999:96:10332-7.

27. Aslam S, Bhattacharya S, Bhattacharya A. The Calmodulin-like calcium binding protein EhCaBP3 of Entamoeba histolytica regulates phagocytosis and is involved in actin dynamics. PLoS Pathog. 2012;8:e1003055.

28. Sebghati TS, Engle JT, Goldman WE. Intracellular parasitism by Histoplasma capsulatum: fungal virulence and calcium dependence. Science. 2000;290:1368-72

29. SBatanghari JW, Deepe Jr GS, Di Cera E, Goldman WE. Histoplasma acquisition of calcium and expression of CBP1 during intracellular parasitism. Mol Microbiol. 1998;27:531-9.

30. Ma W, Smigel A, Tsai YC, Braam J, Berkowitz GA. Innate immunity signaling: cytosolic $\mathrm{Ca}^{2+}$ elevation is linked to downstream nitric oxide generation through the action of calmodulin or a calmodulin-like protein. Plant Physiol. 2008;148:818-28

31. Su S, Panmanee W, Wilson JJ, Mahtani HK, Li Q, Vanderwielen BD, et al. Catalase (KatA) plays a role in protection against anaerobic nitric oxide in Pseudomonas aeruginosa. PLoS One. 2014;9:e91813.

32. Tian Y, Xing Y, Magliozzo R, Yu K, Bloom BR, Chan J. A commercial preparation of catalase inhibits nitric oxide production by activated murine macrophages: role of arginase. Infect Immun. 2000;68:3015-8.

33. Li Y, Severn A, Rogers MV, Palmer RM, Moncada S, Liew FY. Catalase inhibits nitric oxide synthesis and the killing of intracellular Leishmania major in murine macrophages. Eur J Immunol. 1992;22:441-6.

34. Watabe M, Isogai $Y$, Numazawa S, Yoshida T. Role of c-Myc in nitric oxide-mediated suppression of cytochrome P450 3A4. Life Sci. 2003:74:99-108.

35. Kato S, Esumi H, Hirano A, Kato M, Asayama K, Ohama E. Immunohistochemical expression of inducible nitric oxide synthase (iNOS) in human brain tumors: relationships of iNOS to superoxide dismutase (SOD) proteins (SOD1 and SOD2), Ki-67 antigen (MIB-1) and p53 protein. Acta Neuropathol. 2003;105:333-40.

36. Park YJ, Yoon SJ, Suh HW, Kim DO, Park JR, Jung H, et al. TXNIP deficiency exacerbates endotoxic shock via the induction of excessive nitric oxide synthesis. PLoS Pathog. 2013;9:e1003646.

37. Hughes KJ, Meares GP, Chambers KT, Corbett JA. Repair of nitric oxide-damaged DNA in beta-cells requires JNK-dependent GADD45alpha expression. J Biol Chem. 2009;284:27402-8.

38. Takemura Y, Fukuo K, Yasuda O, Inoue T, Inomata N, Yokoi T, et al. Fas signaling induces Akt activation and upregulation of endothelial nitric oxide synthase expression. Hypertension. 2004;43:880-4.

39. Mardente S, Zicari A, Consorti F, Mari E, Di Vito M, Leopizzi M, et al. Cross-talk between NO and HMGB1 in lymphocytic thyroiditis and papillary thyroid cancer. Oncol Rep. 2010;24:1455-61.

40. Gross TJ, Kremens K, Powers LS, Brink B, Knutson T, Domann FE, et al. Epigenetic silencing of the human NOS2 gene: rethinking the role of nitric oxide in human macrophage inflammatory responses. J Immunol. 2014;192:2326-38

41. Dikalov SI, Dikalova AE, Mason RP. Noninvasive diagnostic tool for inflammation-induced oxidative stress using electron spin resonance spectroscopy and an extracellular cyclic hydroxylamine. Arch Biochem Biophys. 2002:402:218-26.

42. Kim JS, Yeo S, Shin DG, Bae YS, Lee JJ, Chin BR, et al. Glycogen synthase kinase 3beta and beta-catenin pathway is involved in toll-like receptor 4-mediated NADPH oxidase 1 expression in macrophages. FEBS J. 2010;277:2830-7.

43. Idelman G, Smith DL, Zucker SD. Bilirubin inhibits the up-regulation of inducible nitric oxide synthase by scavenging reactive oxygen species generated by the toll-like receptor 4-dependent activation of NADPH oxidase. Redox Biol. 2015;5:398-408.

44. Chow CW, Grinstein S, Rotstein OD. Signaling events in monocytes and macrophages. New Horiz. 1995;3:342-51.

45. Zhou X, Yang W, Li J. Ca $\mathrm{Ca}^{2+}$ - and protein kinase C-dependent signaling pathway for nuclear factor-kappaB activation, inducible nitric-oxide synthase expression, and tumor necrosis factor-alpha production in lipopolysaccharide-stimulated rat peritoneal macrophages. J Biol Chem. 2006:281:31337-47.

46. Jeandroz S, Lamotte O, Astier J, Rasul S, Trapet P, Besson-Bard A, et al. There's more to the picture than meets the eye: nitric oxide cross talk with $\mathrm{Ca}^{2+}$ signaling. Plant Physiol. 2013;163:459-70.

47. De Lorenzo BH, Godoy LC, Brito RR N e, Pagano RL, Amorim-Dias MA, Grosso DM, et al. Macrophage suppression following phagocytosis of apoptotic neutrophils is mediated by the s100a9 calcium-binding protein. Immunobiology. 2010;215:341-7.

48. Azenabor AA, Kennedy P, York J. Free intracellular Ca2+ regulates bacterial lipopolysaccharide induction of iNOS in human macrophages. Immunobiology. 2009;214:143-52.

49. Serghides L, McDonald CR, Lu Z, Friedel M, Cui C, Ho KT, et al. PPARy agonists improve survival and neurocognitive outcomes in experimental cerebral malaria and induce neuroprotective pathways in human malaria. PLoS Pathog. 2014;10:e1003980.

50. Kuerban M, Naito M, Hirai S, Terayama H, Qu N, Musha M, et al. Involvement of Fas/Fas-L and Bax/BCl-2 systems in germ cell death following immunization with syngeneic testicular germ cells in mice. J Androl. 2012;33:824-31.

51. Kádár B, Gombos K, Szele E, Ember I, Iványi JL, Csejtei R, et al. Effects of isoflurane on Nfkb p65, Gadd45a and Jnk1 expression in the vital organs of CBA/CA mice. In Vivo. 2011;25:241-4 
52. Qin L, Crews FT. Chronic ethanol increases systemic TLR3 agonist-induced neuroinflammation and neurodegeneration. J Neuroinflammation. 2012;9:130.

53. Diaz-Chavez J, Hernandez-Pando R, Lambert PF, Gariglio P. Down-regulation of transforming growth factor-beta type II receptor (TGF-betaRII) protein and mRNA expression in cervical cancer. Mol Cancer. 2008;7:3.

54. Lu P, Li L, Liu G, Baba T, Ishida Y, Nosaka M, et al. Critical role of TNF-a-induced macrophage VEGF and iNOS production in the experimental corneal neovascularization. Invest Ophthalmol Vis Sci. 2012;53:3516-26.

55. Hsiao PJ, Hsieh TJ, Kuo KK, Hung WW, Tsai KB, Yang CH, et al. Pioglitazone retrieves hepatic antioxidant DNA repair in a mice model of high fat diet. BMC Mol Biol. 2008;9:82.

56. Fang $\mathrm{S}$, Jin $\mathrm{Y}$, Zheng $\mathrm{H}$, Yan J, Cui Y, Bi H, et al. High glucose condition upregulated Txnip expression level in rat mesangial cells through ROS/MEK/ MAPK pathway. Mol Cell Biochem. 2011;347:175-82.

57. Lamas B, Goncalves-Mendes N, Nachat-Kappes R, Rossary A, Caldefie-Chezet F, Vasson MP, et al. Leptin modulates dose-dependently the metabolic and cytolytic activities of NK-92 cells. J Cell Physiol. 2013;228:1202-9.

\section{Submit your next manuscript to BioMed Central and take full advantage of:}

- Convenient online submission

- Thorough peer review

- No space constraints or color figure charges

- Immediate publication on acceptance

- Inclusion in PubMed, CAS, Scopus and Google Scholar

- Research which is freely available for redistribution 